\title{
Prevalência do abuso e da dependência de álcool em Rio Grande (RS): um estudo transversal de base populacional
}

\author{
Newton Luiz Numa Peixoto Primo* \\ Airton Tetelbom Stein**
}

\section{INTRODUÇÃO}

O consumo de álcool é reconhecido como um importante problema de saúde pública ${ }^{1,2}$.

A proporção de dependentes de álcool em relação ao uso na vida, segundo o $\mathrm{CEBRID}^{3}$, é de $20 \%$ para homens e $10 \%$ para mulheres. Nos Estados Unidos, o gasto com o uso de tabaco, álcool e outras drogas atinge 238 bilhões de dólares por ano, sendo 98,6 bilhões gastos com o uso de álcool ${ }^{4}$.

A expectativa de vida de alcoolistas nos Estados Unidos diminui em média 15 anos, sendo as causas de morte, em ordem decrescente: doença cardiovascular, câncer, acidentes de trânsito e suicídio; enquanto isso, o número de internações hospitalares por problemas relacionados ao consumo de álcool alcança $32 \%{ }^{4}$. Nesse mesmo país, a

* Professor Adjunto de Clínica Médica, Departamento Medicina Interna, Fundação Universidade de Rio Grande, RS.

** Professor Titular de Epidemiologia da Faculdade Federal de Ciências Médicas de Porto Alegre e do Curso de Pós-Graduação em Saúde Coletiva da Universidade Luterana do Brasil. Assistente de Coordenação de Ensino do Grupo Hospitalar Conceição. prevalência de alcoolismo atinge 5 a $10 \%^{4}$; na América Latina, é de 3 a $23 \%^{5}$; no Brasil, de 3,4 a $9 \% \%^{1,3,5}$; e em Pelotas (RS), a prevalência é de $9 \%$.

Os objetivos do presente estudo foram: identificar a prevalência de abuso e dependência de álcool na zona urbana de Rio Grande (RS) em indivíduos de 12 a 75 anos de idade; e evidenciar associações existentes entre fatores biológicos, demográficos, socioeconômicos e comportamentais e abuso e dependência de álcool.

\section{MATERIAL E MÉTODOS}

O delineamento utilizado foi do tipo transversal de base populacional com indivíduos de 12 a 75 anos moradores da cidade de Rio Grande.

Utilizou-se o programa Epi-Info para os cálculos de tamanho de amostra, definindo-se como parâmetros básicos um poder estatístico de $80 \%$, prevalência de doença de $9 \%$ (com base no estudo de Pelotas ${ }^{6}$ ) e razão de prevalência $(R P)$ de 1,5 . Assim, o tamanho da amostra estimado foi de 1.156 indivíduos. 
Foi aplicado um questionário padronizado e pré-codificado com 86 questões, as quais contemplavam as variáveis em estudo, nas quais se incluíam o SRQ-20 (Self-Report Questionnaire) e o CAGE.

O SRQ-20 é um questionário que contém 20 perguntas de respostas simples (do tipo sim/ não), indicando casos prováveis de transtornos psiquiátricos menores. O ponto de corte para a positividade foi de cinco ou mais respostas afirmativas para homens e de sete ou mais para mulheres.

O CAGE é um questionário utilizado para estimar a magnitude do alcoolismo em empresas ou populações, e consta de quatro questões básicas a respeito da ingestão de álcool: C (cut-down - diminuir a ingesta), A (annoyed - irritado), G (guilty - culpado), E (eyeopener - identificação de ressaca). O ponto de corte adotado para a positividade do teste foi de duas ou mais respostas positivas.

As variáveis independentes estudadas foram: gênero, cor da pele, idade, classe social, escolaridade, renda, tabagismo, consumo de álcool, café, chimarrão, obesidade, padrão de atividade física, distúrbios psiquiátricos menores, história familiar de consumo de álcool, procedência, situação laboral e ocupação.

As variáveis dependentes foram abuso de álcool e dependência de álcool. O abuso de álcool foi caracterizado como ingestão diária de 30 gramas ou mais para homens e 24 gramas ou mais para mulheres ${ }^{7}$. A dependência de álcool foi definida por duas ou mais respostas positivas ao CAGE.

O perfil sociodemográfico foi avaliado pelo questionário CCEB (Critério de Classificação Econômica Brasil) da Associação Brasileira de Institutos de Pesquisa de Mercado (ABIPEME), que estima o poder de compra das pessoas $\mathrm{e}$ das famílias urbanas, diferenciando-as em classes econômicas.

A obesidade foi aferida através do índice de massa corporal acima de $30 \mathrm{~kg} / \mathrm{m}^{2}$. Foram sorteadas 25 zonas censitárias (conglomerados), sendo entrevistadas 1.044 pessoas. Domicílios onde houve recusa ou nenhum morador foi encontrado, e também aqueles onde os indivíduos da faixa etária em estudo estavam ausentes na primeira visita foram visitados novamente. As recusas e os domicílios de pessoas de difícil acesso não foram substituídos, sendo considerados como perdas. Os domicílios coletivos, como, por exemplo, hotéis, cadeias e escolas, foram excluídos do estudo.

Foram elaboradas duas digitações comparadas no programa Epi-Info, com o objetivo de checagem de consistência. Os erros detectados foram buscados no questionário de origem e retificados na digitação com menor densidade de falhas.

A análise univariada foi descritiva; a bivariada usou o teste do qui-quadrado; a medida de efeito foi RP. Na análise multivariada, foi utilizada regressão logística, tendo-se por medida de efeito a razão de chances (RC) e o intervalo de confiança (IC). Foi considerado estatisticamente significativo $\mathrm{p}<0,05$.

Os dados obtidos foram considerados sigilosos, e os entrevistados apresentaram consentimento informado.

\section{RESULTADOS}

A amostra estudada foi composta por 1.044 pessoas, e as perdas alcançaram $9,7 \%$. Houve predomínio de pessoas de cor branca, que moravam com companheiro, do sexo feminino e da classe social $C$. No que diz respeito à escolaridade (em anos de estudo), um terço das pessoas desta amostra superaram 0 primeiro grau da atual estrutura escolar formal. Referiram haver nascido em Rio Grande 56\% das pessoas estudadas. O desemprego alcançou $20,4 \%$ da população economicamente ativa (PEA) (Tabela 1).

Evidenciou-se que $26 \%$ eram fumantes e, destes, $76 \%$ fumavam mais de 10 cigarros/dia. O consumo de chimarrão atingiu $59,2 \%$ da amostra; destes, $60 \%$ apresentavam este hábito com um padrão diário. O consumo de café foi informado como positivo por 79,5\%, e, destes, $83,7 \%$ o faziam diariamente. O estudo identificou $88,2 \%$ sedentários, $16,2 \%$ obesos e $22,5 \%$ com transtornos psiquiátricos menores.

Aproximadamente um terço da amostra revelava história familiar de consumo de álcool e havia ingerido álcool no último mês. Destes, $24 \%$ beberam cerveja, $4,6 \%$ tomaram vinho e $2,5 \%$ usaram cachaça; $5,5 \%$ abusavam de álcool, e 2,5\% eram dependentes de álcool (Tabela 2).

A análise bivariada evidenciou associação significativa das variáveis dependentes abuso e dependência de álcool com sexo masculino, idade entre 30 e 49 anos, indivíduos pertencentes à classe social $\mathrm{E}$, história familiar positiva de consumo de álcool, consumo de chimarrão e consumo de fumo.

Para controlar fatores de confusão, utilizouse análise multivariada por regressão logística, que manteve a associação existente entre 
Tabela 1 - Descrição da amostra estudada em Rio Grande (RS) - abuso e dependência de álcool

\begin{tabular}{|c|c|c|c|c|c|}
\hline & $\%$ da amostra & № pessoas & $\begin{array}{l}\text { Abuso de } \\
\text { álcool (\%) }\end{array}$ & RP (IC95\%) & $p$ \\
\hline Estado civil & & & & & 0,22 \\
\hline Sem companheiro & 41,20 & 430 & 4,40 & 1,00 & \\
\hline Com companheiro & 58,80 & 613 & 6,20 & $1,40(0,82-2,40)$ & \\
\hline Cor da pele & & & & & 0,48 \\
\hline Brancos & 85,60 & 893 & 5,30 & 1,00 & \\
\hline Não brancos & 14,40 & 150 & 6,70 & $1,27(0,66-2,45)$ & \\
\hline Idade (anos) & & & & & 0,04 \\
\hline 12 a 29 & 35,60 & 371 & 3,50 & 1,00 & \\
\hline 30 a 49 & 37,60 & 392 & 7,60 & $2,18(1,15-4,11)$ & \\
\hline 50 e mais & 26,80 & 279 & 5,00 & $1,43(0,68-2,99)$ & \\
\hline Sexo & & & & & 0,00 \\
\hline Feminino & 59,00 & 615 & 1,60 & 1,00 & \\
\hline Masculino & 41,00 & 428 & 11,00 & $6,76(3,46-13,24)$ & \\
\hline História familiar & & & & & 0,00 \\
\hline Não & 64,40 & 672 & 3,70 & 1,00 & \\
\hline Sim & 35,60 & 371 & 8,60 & $2,31(1,39-3,84)$ & \\
\hline Classe social & & & & & 0,03 \\
\hline$A \in B$ & 20,20 & 210 & 4,50 & 1,00 & \\
\hline$c$ & 46,90 & 489 & 4,30 & $0,96(0,44-2,07)$ & \\
\hline D & 28,20 & 294 & 7,60 & $1,68(0,78-3,58)$ & \\
\hline E & 4,60 & 48 & 13,30 & $2,95(1,11-7,86)$ & \\
\hline Renda familiar (SM) & & & & & 0,20 \\
\hline Mais de 10 & 27,00 & 281 & 5,80 & 1,00 & \\
\hline 6,1 a 10 & 23,50 & 245 & 5,00 & $0,86(0,42-1,79)$ & \\
\hline 3,1 a 6 & 28,00 & 292 & 3,80 & $0,66(0,31-1,40)$ & \\
\hline Até 3 & 21,50 & 224 & 8,20 & $1,42(0,74-2,71)$ & \\
\hline Escolaridade & & & & & 0,50 \\
\hline Mais de 8 anos & 36,20 & 377 & 5,10 & 1,00 & \\
\hline 4 a 8 anos & 53,90 & 562 & 5,70 & $1,12(0,63-1,98)$ & \\
\hline Até 3 anos & 10,00 & 104 & 8,20 & $1,61(0,72-3,59)$ & \\
\hline Procedência & & & & & 0,10 \\
\hline Zona urbana - Rio Grande & 56,10 & 585 & 4,40 & 1,00 & \\
\hline Outra & 43,90 & 458 & 6,80 & $1,53(0,92-2,53)$ & \\
\hline Situação laboral & & & & & 0,49 \\
\hline Trabalhando & 79,60 & 831 & 6,80 & 1,00 & \\
\hline Desempregado & 20,40 & 212 & 4,90 & $0,72(0,29-1,83)$ & \\
\hline Ocupação & & & & & 0,08 \\
\hline Proprietário/nível superior & 8,30 & 86 & 9,60 & 1,00 & \\
\hline Especializado/semi-especializado & 21,10 & 220 & 6,70 & $0,69(0,30-1,59)$ & \\
\hline Não-qualificado & 8,90 & 92 & 7,90 & $0,82(0,31-2,15)$ & \\
\hline Fora da PEA* & 61,60 & 643 & 4,10 & $0,42(0,20-0,91)$ & \\
\hline
\end{tabular}

$n=1044$

$\mathrm{RP}=$ razão de prevalência; $\mathrm{IC} 95 \%$ = intervalo de confiança de 95\%; SM = salário mínimo; PEA = população economicamente ativa. 
Tabela 2 - Análise bivariada para identificação de fatores de risco e associação com alcoolismo (Rio Grande, RS)

\begin{tabular}{lccccc}
\hline & Alcoolismo & No pessoas & $\begin{array}{c}\text { Sem } \\
\text { alcoolismo (\%) }\end{array}$ & RC & p \\
\hline $\begin{array}{l}\text { Tabagismo } \\
\quad \text { Não }\end{array}$ & 62,50 & 652 & 2,10 & 1,00 & 0,00 \\
$\quad$ Ex-fumante & 11,30 & 117 & 10,20 & $4,74(2,25-9,98)$ & \\
$\quad$ Sim & 26,20 & 273 & 11,30 & $5,27(2,85-9,75)$ & \\
Número de cigarros/dia & & & & & 0,34 \\
$\quad$ Até 9 & 23,80 & 248 & 14,30 & 1,00 & \\
$\quad$ 10 a 19 & 29,80 & 311 & 7,90 & $0,55(0,25-1,23)$ & \\
$\quad$ 20 e mais & 46,30 & 483 & 11,30 & $0,79(0,41-1,52)$ & \\
Consumo diário de café & & & & & 0,13 \\
$\quad$ Não & 16,30 & 170 & 8,90 & 1,00 & \\
$\quad$ Sim & 83,70 & 873 & 5,50 & $0,62(0,33-1,15)$ & \\
Consumo diário de chimarrão & & & & & 0,03 \\
$\quad$ Não & 40,00 & 417 & 4,00 & 1,00 & \\
$\quad$ Sim & 60,00 & 626 & 8,60 & $2,13(1,07-4,25)$ & \\
Sedentarismo & & & & & 0,17 \\
$\quad$ Não & 11,80 & 123 & 8,10 & 1,00 & \\
$\quad$ Sim & 88,20 & 920 & 5,10 & $0,63(0,33-1,21)$ & \\
Obesidade & & & & & 0,13 \\
$\quad$ Não & 83,80 & 874 & 5,90 & 1,00 & \\
$\quad$ Sim & 16,20 & 169 & 3,00 & $0,51(0,21-1,25)$ & \\
Distúrbios psiquiátricos menores & & & & & 0,79 \\
$\quad$ Não & 77,50 & 809 & 5,60 & 1,00 & \\
$\quad$ Sim & 22,50 & 234 & 5,10 & $0,92(0,49-1,71)$ & \\
\hline
\end{tabular}

$n=1044$

$\mathrm{RC}=$ razão de chances.

abuso e dependência de álcool e as variáveis sexo masculino $(R C=6,28)$, fumante $(R C=5,42)$ e classe social $E(R C=5,37)$ (Tabela 3$)$.

\section{DISCUSSÃO}

Os estudos transversais são largamente utilizados para investigar a prevalência de condições, permitindo melhorar o planejamento das ações de saúde e gerar hipóteses de novos estudos ${ }^{1,5,6,8,9}$.

A distribuição desta amostra por cor da pele, sexo, estado civil e transtornos psiquiátricos menores é semelhante às distribuições encontradas em outros estudos realizados em Pelotas ${ }^{6}$, Porto Alegre $(\mathrm{RS})^{7}$ e no Rio de Janeiro (RJ) ${ }^{10,11}$.

O consumo de álcool encontrado em amostras populacionais nos Estados Unidos $(90 \%)$ e na Austrália $(87 \%)$ mostram valores semelhantes. O consumo apresentado no Canadá (75\%), embora inferior aos já citados, é bastante superior ao da Colômbia e ao do México (51\%), que é comparável ao do Rio de Janeiro $(52 \%)^{10}$. O consumo revelado neste estudo aponta para uma proporção surpreendentemente inferior (33\%).

O consumo abusivo de álcool observado em um estudo realizado em Porto Alegre $(15 \%)^{11}$ foi superior ao de Pelotas $(9 \%)^{6,11}$ e de Rio Grande (5,5\%).

A dependência de álcool em Rio Grande, que corresponde a $2,5 \%$ (evidenciada pelo $\left.\mathrm{CAGE}^{4,12-18}\right)$, mostra-se significativamente inferior à encontrada nos Estados Unidos $(5 \%$ entre mulheres e $10 \%$ entre homens) e em Porto Alegre $\left(9 \%^{7}\right)$, e é bastante similar aos valores encontrados em Pelotas $\left(4 \%^{6,11}\right)$ e no Rio de Janeiro $\left(3 \%{ }^{10}\right)$.

A disparidade para menos na proporção de 
Tabela 3 - Análise multivariada para identificação de fatores de risco e associação com alcoolismo (Rio Grande, RS)

\begin{tabular}{|c|c|c|}
\hline & RC (IC95\%) & $p$ \\
\hline História familiar & & 0,3 \\
\hline Não & 1 & \\
\hline Sim & $1,82(0,93-3,55)$ & \\
\hline Classe social & & 0,02 \\
\hline$A e B$ & 1 & \\
\hline C & $0,72(0,25-2,03)$ & \\
\hline D & $1,13(0,40-3,22)$ & \\
\hline$E$ & $5,37(1,31-21,96)$ & \\
\hline Idade/anos & & 0,26 \\
\hline $12-29$ & 1 & \\
\hline $30-49$ & $1,79(0,69-4,64)$ & \\
\hline 50 e mais & $0,98(0,32-2,99)$ & \\
\hline Sexo & & 0,00 \\
\hline Feminino & 1 & \\
\hline Masculino & $6,28(2,82-14,00)$ & \\
\hline Fumo & & 0,001 \\
\hline Não & 1 & \\
\hline Ex-fumante & $3,36(1,05-10,75)$ & \\
\hline Sim & $5,42(2,18-13,49)$ & \\
\hline Chimarrão & & 0,31 \\
\hline Consumo não diário & 1 & \\
\hline Consumo diário & $1,51(0,68-3,37)$ & \\
\hline
\end{tabular}

$\mathrm{n}=1044$

$\mathrm{RC}=$ razão de chances; IC95\% = intervalo de confiança de 95\%.

consumo e abuso de álcool em Rio Grande quando comparada aos demais estudos poderia ser explicada pelas perdas ocorridas nesta pesquisa, na hipótese de que os nãorespondentes apresentassem proporções de consumo de álcool superiores àqueles que responderam ao questionário. Entretanto, existe a possibilidade de que este achado reflita a realidade ${ }^{19,20}$.

O presente estudo evidenciou uma prevalência de abuso e dependência de álcool aproximadamente duas vezes menor em Rio Grande do que em Pelotas. Consideradas as limitações, os resultados deste estudo mostram uma proporção inferior de positividade ao CAGE em relação a estudos realizados em Pelotas, Porto Alegre, Rio de Janeiro e nos Estados Unidos. Uma possível interpretação para essa variação pode ser a magnitude das perdas, com $9,7 \%$ de taxa de não-respostas no estudo (viés
Entre as variáveis estudadas, seis exerceram efeito sobre 0 abuso e a dependência de álcool: uma do tipo socioeconômica (classe social E), três do tipo biológico (idade de 30 a 49 anos, sexo masculino, história familiar de consumo) e duas do tipo comportamental (tabaco e chimarrão). A associação entre tabagismo e abuso e dependência de álcool é confirmada pela literatura $3,8,11,21,22-25$. Entretanto, devido ao fato de exposição e desfecho terem sido coletados em um mesmo momento, torna-se necessário chamar a atenção para a possível existência de causalidade reversa.

Após proceder à análise multivariada, as variáveis história familiar de consumo de álcool, idade e consumo de chimarrão deixaram de apresentar associação significativa, e as variáveis sexo, tabaco e classe social E mantiveram efeito sobre abuso e dependência de álcool. 


\section{CONCLUSÃO}

A execução desta pesquisa evidenciou as dificuldades de se conduzir um estudo de base populacional sobre alcoolismo. Estimou-se que, em Rio Grande, 5,5\% da população abusa de álcool e $2,5 \%$ é dependente. O grupo formado por homens fumantes e de baixo nível socioeconômico é mais vulnerável ao abuso e à dependência de álcool. Os serviços de saúde de Rio Grande devem desenvolver campanhas para diminuir o uso de álcool, com especial atenção nesse estrato da população.

\section{REFERÊNCIAS BIBLIOGRÁFICAS}

1. Cyr MG, Wartman S. The effectiveness of routine screening questions in the detection of alcoholism. JAMA 1988;259:51-4

2. Ettner SL. Measuring the human cost of a weak economy: does unemployment leads to alcohol abuse? Soc Sci Med 1997;44:251-60.

3. Carlini EA, Galduroz JE, Nappo SA e Secretaria Nacional Antidrogas e Centro Brasileiro de Informações sobre Drogas Psicotrópicas (CEBRID). I Levantamento domiciliar sobre uso de drogas no Brasil. São Paulo: Cromosste; 2001. p.303-4.

4. Lewis DC The role of the generalist in the care of substance abusing patient. Med Clin N Am 1997;81:83143.

5. Mariategui JS. Investigación epidemiológica del alcoholismo. Rev Assoc Bras Psiquiatr 1985;26:109-13.

6. Lima M. Epidemiologia do alcoolismo. In: Ramos SP, Bertolote JM. Alcoolismo hoje. $3^{\underline{a}}$ ed. Porto Alegre: Artes Médicas; 1997. p.45-65.

7. Moreira LB, Fuchs FD, Moraes RS, Bredemeier M, Cardozo S, Fuchs SC, et al. Alcoholic beverage consumption and associated factors in Porto Alegre, a southern Brazilian city: a population-based survey. J Stud Alcohol 1996;57:253-9.

8. Bobo JK. Nicotine dependence and alcoholism epidemiology and treatment. J Psychoactive Drugs 1992;24:123-9.

9. Batel PP, Maitre F, Rueff C. Relationship between alcohol and tobacco dependencies among alcoholics who smoke. Addiction 1995;90:977-80.

10. Almeida LM, Coutinho E. Prevalência de consumo de bebidas alcoólicas e de alcoolismo em uma região metropolitana do Brasil. Rev Saude Publica 1993;27:23-9.

11. Lima M. Fatores associados ao consumo de álcool. In Ramos SP, Bertolote JM. Alcoolismo hoje. $3^{\underline{a}}$ ed. Porto Alegre: Artes Médicas; 1997. p. 53-63.

12. Masur J, Monteiro MG. Validation of the CAGE alcoholism screening test in a Brazilian psychiatric inpatient hospital setting. Braz J Med Biol Res 1983;16:215-8.

13. Mayfield D, McLeod G, Hall P. The CAGE questionnaire: validation of a new alcoholism screening instrument. Am J Psychiatry 1974;131:1121-3.

14. Saunders WM, Kershaw P. Screening tests for alcoholism findings of a community study. Br J Addiction 1980;75:3741.

15. Mari JJ. A validity study of a psychiatric screening questionnaire (SRQ 20) in primary care in the city of São Paulo. Br J Psychiatry 1986;148:23-6.

16. Lester D. Suicide, alcohol and divorce: a comment. Addiction 1995;90:985-90.
17. Ewing J. Detecting alcoholism: the CAGE questionnaire. JAMA 1984;252:1905-6.

18. Ewing J. Recognizing, confronting and helping the alcoholic. Am Fam Physician 1978;18:107-14.

19. Santana VS, Almeida FN. Alcoolismo e consumo de álcool: resumo de achados epidemiológicos. Rev ABPAPAL 1987;9:15-22.

20. Soibelman M, Luz E, Diemen LV. Problemas relacionados ao consumo de álcool. In: Duncan BB. Medicina ambulatorial: condutas clínicas em atenção primária. $2^{a}$ ed. Porto Alegre: Artes Médicas; 2004. p.539-49.

21. Schorling JB, Buschbaum DG. Screening for alcohol and drug abuse. Med Clin N Am 1987;81:831-43.

22. Chou SP. Sex differences in morbidity among respondents classified as alcohol abusers and or dependent: results of a national survey. Addiction 1994;89:87-93.

23. Lemmens $\mathrm{PH}$. The alcohol content of self-report and standard drinks. Addiction 1994;89:593-601.

24. Lieber CS. Medical disorders of alcoholism. N Engl J Med 1995;333:1052-8

25. Stein MD, Cyr MG. Women and substance abuse. Med Clin N Am 1997;81:979-98.

\section{RESUMO}

Introdução: O consumo de álcool é um problema de saúde pública muito relevante. O objetivo do presente estudo foi identificar a prevalência de alcoolismo e explorar possíveis fatores que contribuam para esse hábito. Metodologia: Foi realizado um estudo transversal de base populacional no município de Rio Grande (RS), constando de uma amostra de 1.044 indivíduos, de ambos sexos, com idade entre 12 e 75 anos. Definiu-se dependência de álcool pelo questionário CAGE, e o abuso de álcool foi caracterizado pelo consumo de 30 ou mais gramas por dia para homens e 24 ou mais gramas por dia para mulheres. Para controlar o efeito de fatores de confusão, procedeu-se a uma análise multivariada por regressão logística. Resultados: $5,5 \%$ dos indivíduos abusavam de álcool, dos quais 2,5\% eram dependentes. Após controle por possíveis fatores de confusão, identificou-se uma associação significativa entre alcoolismo e as seguintes variáveis: sexo masculino (razão de chances - $R C=6,28$ ), tabagismo $(R C=5,42)$ e classe social $E(R C=5,37)$. Conclusão: Os resultados identificaram as dificuldades de se realizar um estudo de base populacional sobre alcoolismo e revelaram que homens que fumam e são de classe social mais baixa têm uma chance maior de apresentar problemas vinculados ao álcool. A prevalência de abuso de álcool em Rio Grande foi de $5,5 \%$, e de dependência, $2,5 \%$.

Descritores: Álcool, abuso, dependência, epidemiologia, estudo transversal, atenção primária.

\section{ABSTRACT}

Introduction: Alcohol consumption is serious public health issue. This study aimed at determining the prevalence of alcohol consumption in a southern 
Brazilian municipality and at investigating associated factors. Methods: A cross-sectional populationbased survey was carried out in the municipality of Rio Grande, southern Brazil, with a sample of 1,044 people, both male and female, aged 12 to 75 years. Alcohol dependence was defined according to the CAGE questionnaire, and alcohol abuse was defined as the daily consumption of at least 30 grams of alcohol for men and 24 grams for women. Potential confounding factors were controlled in the multivariate analysis (logistic regression). Results: Alcohol abuse was found in $5.5 \%$ of the sample, and dependence in $2.5 \%$. After adjusting for confounding factors, a significant association was observed between alcohol consumption and the following variables: male gender (odd ratio - OR=6.28), smoking $(O R=5.42)$, and social class $E(O R=5.37)$. Conclusion: Our results revealed the difficulties involved in the performance of a population-based study to assess alcohol consumption and showed that men who smoke and come from lower-income groups have greater chances of presenting alcoholrelated problems. The prevalence of alcohol abuse in Rio Grande was of $5.5 \%$, and of alcohol dependence, of $2.5 \%$.

Keywords: Alcohol, abuse, dependence, epidemiology, cross-sectional study, primary care. Title: Prevalence of alcohol abuse and dependence in Rio Grande, state of Rio Grande do Sul: a crosssectional, population-based survey

\section{RESUMEN}

Introducción: El consumo de alcohol es un problema de salud pública muy relevante. El objetivo de este estudio fue identificar la prevalencia de alcoholismo y explorar posibles factores de riesgo. Metodología: Se hizo un estudio transversal de base poblacional en Rio Grande (RS), con una muestra de 1.044 individuos de ambos sexos, con edades entre 12 y 75 años. La dependencia de alcohol fue definida por el cuestionario CAGE, y el abuso de alcohol fue caracterizado por el consumo de 30 o más gramos / día para hombres y 24 gramos / día para mujeres. Para controlar el efecto de factores de confusión, se hizo un análisis multivariado por regresión logística. Resultados: El 5,5\% de los individuos abusaban del alcohol, siendo el 2,5\% dependientes. Después del control por posibles factores de confusión, se identificó una asociación significativa entre el alcoholismo y las siguientes variables: sexo masculino (razón de chances $-R C=6,28)$, tabaquismo $(R C=5,42)$ y clase social $E(R C-5,37)$. Conclusión: Los resultados identificaron las dificultades de realizar un estudio de base poblacional sobre alcoholismo y revelaron que hombres que fuman y pertenecen a clases sociales más bajas tienen una mayor posibilidad de presentar problemas vinculados al alcohol.

Palabras clave: Alcohol, abuso, dependencia, epidemiología, estudio transversal, atención primaria. Título: Prevalencia del abuso y de la dependencia de alcohol en Rio Grande (RS): un estudio transversal de base poblacional

Correspondência:

Newton Luiz Numa Peixoto Primo

Rua Dom Bosco, 26/301 - Bairro Cidade Nova

CEP 96211-090 - Rio Grande - RS

E-mail:primo@mikrus.com.br

Copyright (C) Revista de Psiquiatria do Rio Grande do Sul - SPRS 